\title{
Quantitative Forecast of Daily and Monthly Pedestrian Flows in the Scenic Spot
}

\author{
J.Y.Wang, W.G.Weng \& Y.Zhou \\ Institute of Public Safety Research, Department of Engineering Physics, Tsinghua University, Beijing, China
}

\begin{abstract}
Study on the pedestrian flow forecasting and pre-warning has scientific value and practical significance to organize crowd activities and decrease stampede risk. In this paper, the changing rules of daily and monthly pedestrian flows are analyzed, taking AQI (Air Quality Index) into consideration. The correlations of pedestrian flows, including daily and monthly pedestrian flows, and affecting factors are quantitatively investigated. The simulation equations established in this paper are reliable and accurate to forecast the daily and monthly pedestrian flows. The results can help to conduct security pre-warning system and safety scheme guide for crowd activities.
\end{abstract}

KEYWORD: pedestrian flows; forecasting; AQI

\section{INSTRUCTIONS}

With the development of economic, the recent increase in the frequency of large-scale mass activities is dramatically high. According to incomplete statistics, thousands of people were killed by stampede in highly dense crowd around the world (Schadschneider et al. 2009). This arouses serious safety issues and tough tasks for organization of mass events and emergency management. It has scientific value and practical significance to study pedestrian flow forecasting, pre-warning and emergency response.

Rosselló-Nadal et al. (2011) used transfer function models to investigate the effect of the shortterm weather conditions on British outbound flows. The weather variables, including mean temperature, heat waves, air frost and sunshine days, were analyzed and proved significantly related to the dynamics of the outbound British flows. Song et al. (2012) developed a tourism demand forecasting system for Hong Kong based on the web techniques. The system generated the forecasts of tourist arrivals, tourist expenditure, demand for hotel rooms, sectoral demand and outbound tourist flows. An overall investigation of 183 important scenic spots in Beijing was made to explore the overall trend as well as the distributing character of visitor flow in scenic spots. Some problems, including the imbalance of spacial and temporal distribution of the visitor flow in Beijing and insufficient supply of the tourism products, were found (Niu et al. 2005). Liu et al. (2014) developed an optimization model based on GM $(1,1)$ model. The prediction accuracy of the model was verified by numerical example. Support vector regression optimized by genetic algorithm (GSVR) is proposed to forecast tourism demand. The feasibility of SVR was examined by comparing it with back-propagation neural networks (BPNN) (Cai et al. 2009). A model, combining Support Vector Regression with Adaptive Particle Swarm Optimization, was constructed to forecast short-term tourism flow. The daily data set of a 5A mountain resorts from 2008 to 2011 summer holidays in Mount Huangshan is applied as an example (Chen et al. 2013). Based on the numeralized climate comfort degree and fictitious factors, the correlation of monthly index and climate comfort degree was found by the method of OLS (Ma et al. 2009). The tourism security pre-warning system in China was proposed and divided into four models, including emergency warning model, environmental pollution warning model, tourism capacity warning model and tourism business warning model. The tourism capacity warning model can help to prevent the occurrence of excessive crowd and even stampede (Xie et al. 2010). But most of the researches are about annual pedestrian flows forecasting. The study on the forecast and pre-warning of monthly and daily pedestrian flows is insufficient. And the influence of AQI (Air Quality Index) on the monthly and daily pedestrian flows has been neglected.

In this paper, the correlations of pedestrian flows, including daily and monthly pedestrian flows, and affecting factors are quantitatively investigated. In Section 2, the data of pedestrian flows in the scenic 
spot in Beijing is used to analyze by linearregression analysis method. Then in Section 3, the characteristics of pedestrian flows are investigated and the simulation equations are established, followed by the conclusion section.

\section{MATERIALS AND METHOD}

As the political and cultural center of China, Beijing has a high population density and attracts large number of travelers. The significances of public safety are indubitable. The occurrence of Rainbow Bridge crowd disaster has increased the public's awareness of public safety and more and more researchers become interested in the study of risk prevention and control methods for stampede in highly dense crowd. In this paper, the data of pedestrian flows in one scenic spot in Beijing is used to analyze the characteristics of pedestrian flows and the correlations with affecting factors. The time interval of the data is from January 1, 2013 to December 31,2013 . The daily pedestrian flows are affected by many factors, such as the weather, wind velocity, the temperature, AQI (Air Quality Index) and so on. But concerned with monthly pedestrian flows, the monthly averaged weather factor has not any practical meanings. The temperature-humidity index is adopted instead. The temperature-humidity index is an indicator of climate comfortable degree. It can reflect heat exchange between human body and the surrounding environment by the combination of temperature and humidity.

$$
T H I=(1.8 t+32)-0.55(1-f)(1.8 t-26)
$$

where THI represents temperature-humidity index, $t$ represents temperature and its unit is ${ }^{\circ} \mathrm{C}, f$ represents relative humidity.

In this paper, the relationships between daily and monthly pedestrian flows and their respective affecting factors were investigated by linearregression analysis method.

\section{RESULTS AND DISCUSSIONS}

\subsection{The daily pedestrian flows}

Based on the data set of daily pedestrian flows from January 1, 2013 to December 31, 2013 in one scenic spot in Beijing, we can study the characteristics of daily pedestrian flows. Figure 1 shows the timedependent daily pedestrian flows in a whole year from January 1 to December 31, 2013. Obviously, there are some anomalous points around 1, 44, 94, 121 and 274 day, which respectively correspond to New Year's Day, Spring Festival, Tomb-sweeping Festival, International Labor Day and National Day. We can easily find that the statutory holidays have a great influence on the daily pedestrian flow. During the National Day, the daily pedestrian flows are all above 200,000. The first two days of the National Day, the daily pedestrian flows even reach 430,000 . Thus we can come to a conclusion that the weight of the National Day is higher than other holidays. Getting rid of these anomalous points, the timedependent daily pedestrian flows are distributed in "M" type, shown in figure 1. One of the daily pedestrian flows increase is from April to May when the climate is comfortable, another is from July to August when the Chinese summer vacation is approaching.

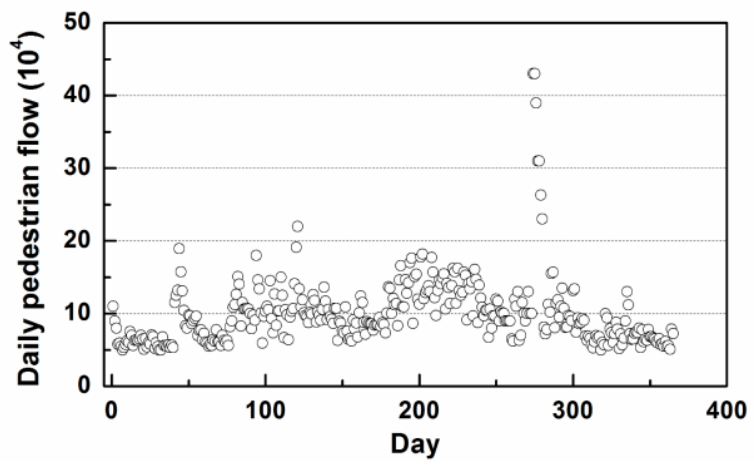

Figure 1. Time series of daily pedestrian flows in a whole year from January 1 to December 31, 2013.

Figure 2 shows the daily pedestrian flows distribution in a whole year from January 1, 2013 to December 31, 2013. The pulse function is used to fit the distribution, as follows.

$$
y=0.26+3.3 \times 10^{7} e^{\frac{1.5-x}{2}}\left(1-e^{\frac{1.5-x}{143.4}}\right)^{3.12}
$$

We can estimate the capacity of the scenic spot in Beijing based on the cumulative distribution as shown in figure 2 . The 50 th percentile value equals 80,000 , meaning that the daily pedestrian flows are below 80,000 in nearly half a year. The 99th percentile value is 300,000 . It means there is little possibility for the daily pedestrian flows to exceed 300,000 . Once the daily pedestrian flow is over 300,000 , we should be on the alert and keep an eye on the real-time pedestrian flow.

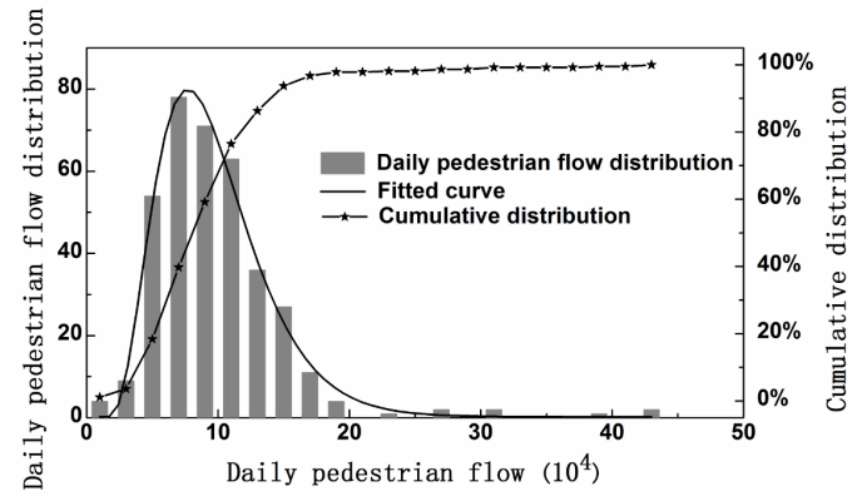

Figure 2. Daily pedestrian flows distribution in a whole year January 1, 2013 to December 31, 2013. The fitted curve is pulse function. 
The daily pedestrian flows are affected by many factors, such as the weather, wind velocity, the temperature, the holiday index and AQI (Air Quality Index). The holiday index represents the influence of statutory holidays on the daily pedestrian flows. Based on the above study, the holiday index of the National Day should be higher than those of others. By Experts Grading method, we can quantify the weather factor and holiday index, shown in table 1 . In table 1, the other statutory holidays include New Year's Day, Spring Festival, Tomb-sweeping Festival and International Labor Day. Then based on the linear-regression analysis method, the relationship between daily pedestrian flows and the corresponding affecting factors is constructed, seen in the equation (3).

Table 1. Affecting factors

\begin{tabular}{|l|l|}
\hline W (Weather) & H (Holiday index) \\
\hline $1 \quad$ rainy or snowy & 1 weekdays \\
\hline 2 cloudy & 2 weekend \\
\hline 4 shiny & 4 other statutory holidays \\
\hline & 8 the National Day \\
\hline
\end{tabular}

$$
\begin{aligned}
Y= & 8.82+0.12 W+0.12 V+0.12 T+0.45 H \\
& -0.00 \$ \mathbb{Q},=\quad 0 .
\end{aligned}
$$

where $Y$ is a representative of daily pedestrian flows. $W$ represents weather conditions and its value can be 1,2 or $4 . V$ is wind velocity and its unit is $m / s . T$ means temperature, its unit is ${ }^{\circ} \mathrm{C}$. $H$ represents holiday index and its value can be 1 , 2, 4 or 8. $A Q$ means Air Quality Index and can be obtained from http://jc.bjmemc.com.cn/AirQualityDaily/DataSearc h.aspx.

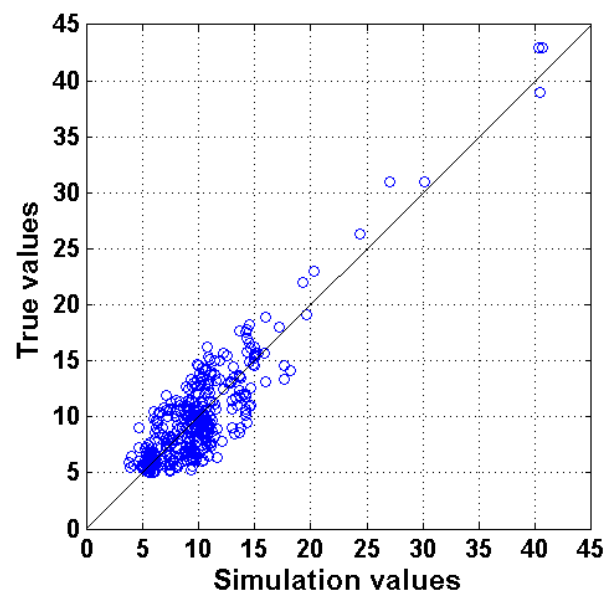

Figure 3. Comparison of simulation values of daily pedestrian flows by linear-regression analysis method and the true values. The reference line is $\mathrm{y}=\mathrm{x}$.

The reliability of the simulation equation was validated by comparison the simulation values of daily pedestrian flows and the true values. As shown in figure 3 , the data points scattered near the reference line $y=x$. The results prove that the simulation equation established in this paper is feasible and reliable.

\subsection{The monthly pedestrian flows}

Figure 4 shows time series of monthly pedestrian flows in a whole year from January to December, 2013. There are three points, whose values exceed 4 million, respectively located in July, August and October. In July and August, the student summer holiday is coming and the monthly pedestrian flows are naturally increasing. The National Day is the longest holiday for all workers and students. Thus the monthly pedestrian flows increase dramatically.

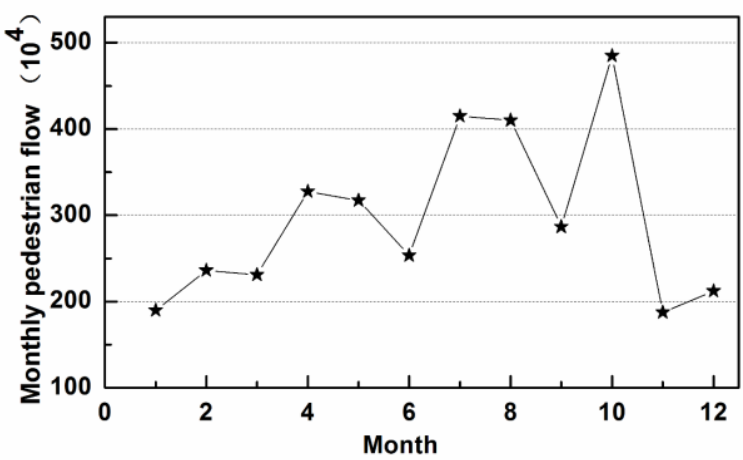

Figure 4. Time series of monthly pedestrian flows in a whole year from January to December, 2013.

Compared with daily pedestrian flows, the affecting factors of monthly pedestrian flows are different. We adopt three factors to analyze their correlation, seen in the equation (4).

$$
\begin{aligned}
& Y=364.97+15.42 * T H I+156.40 * H-0.52 * A Q, \\
& R^{2}=0.9838
\end{aligned}
$$

where $Y$ means monthly pedestrian flows. THI is a representative of temperature-humidity index. $H$ represents holiday index. Its value is 1 in July, August and October and equals 0 in other months. $A Q$ is the monthly averaged Air Quality Index.

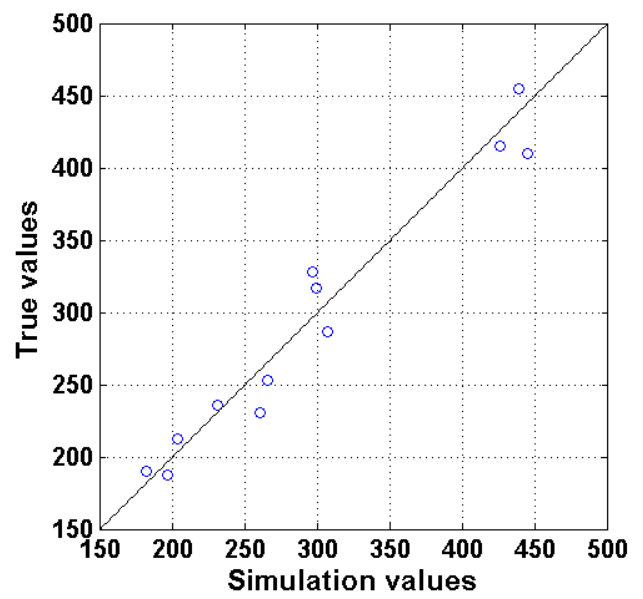

Figure 5. Comparison of simulation values of monthly pedestrian flows by linear-regression analysis method and the true values. The reference line is $\mathrm{y}=\mathrm{x}$. 
Compared with the true values of monthly pedestrian flows, the accuracy of simulation values can be verified. As shown in figure 5, the data points scattered near the reference line $\mathrm{y}=\mathrm{x}$. The results prove that the simulation equation can be adopted to estimate monthly pedestrian flows.

\section{CONCLUSIONS}

In this paper, the correlative analysis on pedestrian flows and corresponding affecting factors is performed quantitatively. Some results about daily and monthly pedestrian flows are presented.

The statutory holidays have a great influence on the daily pedestrian flow and the weight of the National Day is higher than other holidays. The time-dependent daily pedestrian flows are distributed in "M" type, ignoring the holiday points. The capacity of the scenic spot in Beijing is estimated, about 300,000. The relationship between daily pedestrian flows and corresponding affecting factors is investigated and verified. Thus we can forecast the daily pedestrian flows based on the data set of affecting factors. The characteristics and the simulation equation of monthly pedestrian flows are investigated in detail. The results proposed here can help to perfect security pre-warning system and safety scheme guide for crowd activities. Thus we can decrease stampede risk and reduce the injuries and deaths in crowd activities.

\section{ACKNOWLEDGEMENT}

This paper was supported by National Natural Science Foundation of China (Grant No. 71473147), and Tsinghua University Initiative Scientific Research Program (Grant No. 2012THZ02160).

\section{REFERENCES}

[1] Cai, Z.J., Lu, S., Zhang, X. B. 2009.Tourism demand forecasting by support vector regression and genetic algorithm. 2nd IEEE International Conference on Computer Science and Information Technology, Chongqing, 2009: 144-146.

[2] Chen, R., Liang, C.Y., Liang, Y., et al. 2013.Forecasting Short-Term Tourism Flow of Mountain Resorts Based on Adaptive PSO-SVR. Tourism Science 27(3): 50-60.

[3] Liu, X.Y., Peng, H.Q., Bai, Y., et al. 2014.Tourism Flows Prediction based on an Improved Grey GM $(1,1)$ Model. Procedia-Social and Behavioral Sciences 138: 767-775.

[4] Ma, L.J., Sun, G.N., Kang G.D., et al. 2009.Correlative Analysis on Climate Comfortable Degree and Monthly Variations of Tourists in Beijing. Journal of Arid Land Resources and Environment 2: 018.

[5] Niu, Y.F., Xie, L.B. \& Lie, C.F. 2005.The variation tendency and proposed countermeasures for adjusting and controlling visitor flow in Beijing. Geographical Research 2: 283-292.

[6] Rosselló-Nadal, J., Riera-Font, A. \& Cárdenas, V. 2011. The impact of weather variability on British outbound flows. Climatic change 105(1-2): 281-292.

[7] Schadschneider, A., Klingsch, W., Klüpfel, H., Kretz T, Rogsch C. \& Seyfried, A. 2009. Evacuation dynamics: empirical results, modeling and applications. in: Meyers, B. ed. Encyclopedia of Complexity and Systems Science, Springer, Berlin, 2009, pp. 3142-3176.

[8] Song, H.Y., Gao, Z.X., Zhang, X.Y., et al. 2012. A webbased Hong Kong tourism demand forecasting system. International Journal of Networking and Virtual Organisations 10(3-4):275-291.

[9] Xie, C.W. 2010. Study on the construction of tourism security pre-warning system in China. China Safety Science Journal 20(8): 170-176. 